\title{
COMPLETE HYPERBOLIC NEIGHBORHOODS IN ALMOST-COMPLEX SURFACES
}

\author{
R. Debalme*, S. Ivashkovich*,†
}

\section{Introduction.}

Our goal here is to prove that each point in an almost-complex surface has a basis of complete hyperbolic neighborhoods. The problem is local, and therefore we can consider the case when our surface is $\mathbb{R}^{4}$ with an arbitrary almost-complex structure $J$. Throughout this paper, almost-complex structures are assumed to be of class $C^{1, \alpha}$ for some $0<\alpha<1$. Let $C$ be some non-singular $J$-complex curve passing through the origin.

We shall prove the following

Theorem. There exists a basis $\left\{U_{j}\right\}$ of neighborhoods of zero in $\mathbb{R}^{4}$, such that:

1) $\left(U_{j}, J\right)$ are complete hyperbolic in the sence of Kobayashi;

2) $\left(U_{j} \backslash C, J\right)$ are complete hyperbolic as well.

In the case when $J$ is integrable, this result follows easily from the Pick-Schwarz lemma. Indeed, one can choose a local holomorphic coordinate system so that $C$ is one of the axes, and then set $U_{j}=\Delta_{\frac{1}{j}}^{2}$, the bidisk of radius $\frac{1}{j}$. Now the Kobayashi distance on $U_{j}$ and $U_{j} \backslash C$ can be calculated explicitly and one can see from this explicit form that it is complete, see e.g. [Ko-2], Ch.2. The utility of complete hyperbolic neighborhoods in the theory of complex hyperbolic manifolds, first observed by Kiernan and Kobayashi, has become standard since. The fact that this result remains true for any almost-complex structure is somewhat suprising. Really, given any germ of a non-singular real surface $C \ni 0$ in $\mathbb{R}^{4}$, one can easily construct an almost-complex structure $J$ in a neighborhood of zero such that $C$ becomes a $J$-complex curve. Note also that because of the non-existence of $J$ holomorphic functions for general $J$, the hyperbolicity of $U \backslash C$ for a $J$-complex curve $C$ is not evident either.

An open subset $Y$ in an almost-comlex manifold $(X, J)$ is called locally complete hyperbilic (l.c.h.) if for every $y \in \bar{Y}$ there exists a neighborhood $V_{y} \ni y$ such that $V_{y} \cap Y$ is complete hyperbolic. An example of this situation we shall have in mind is when $(X, J)$ is an almost-complex surface and $Y=X \backslash D$, where $D=\cup_{k} D_{k}$ is a (reducible) curve with irreducible $J$-complex components $D_{k}$, which do not have cusps. Our theorem insures that such a $Y$ is l.c.h.

The following proposition is due to Zaidenberg [Za-1] in the case of complex manifolds . Its proof goes through for almost-complex manifolds as well.

* Address: UFR de Mathématiques, Université de Lille 1, 59655 Villeneuve d'Ascq Cedex, France. e-mail: debalme@agat.univ-lille1.fr; ivachkov@agat.univ-lille1.fr

$\dagger$ This work was partially done when the author visited the university of Wisconsin-Madison. He would like to thank this Institution for the hospitality.

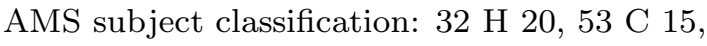

Key words: Kobayashi hyperbolic, Schwarz lemma, almost-complex manifold. 
Proposition. Let $Y$ be a relatively compact l.c.h. open subset of an almostcomplex manifold $(X, J) . Y$ is hyperbolically imbedded into $X$ if and only if $Y$ does not contain $J$-complex lines and admits no limiting $J$-complex lines.

Recall that an open subset $Y$ of an almost-comlex manifold $X$ is called hyperbolically imbedded into $X$ if for any two sequences $\left\{x_{n}\right\},\left\{y_{n}\right\}$ in $Y$ converging to $x \in \bar{Y}$ and $y \in \bar{Y}$, respectively, one has that $\overline{\lim }_{n \rightarrow \infty} k_{Y, J}\left(x_{n}, y_{n}\right)>0$. Here $k_{Y, J}$ denotes the Kobayashi pseudodistance on the manifold $(Y, J)$. It it worth observing that if $Y$ is hyperbolically imbedded into $(X, J)$ and is l.c.h. then $(Y, J)$ is complete hyperbolic, see $[\mathrm{Ki}]$. As in the integrable case, by a $J$-complex line we understand a non-constant $J$-holomorphic map $f: \mathbb{C} \rightarrow X$ such that $\left\|d_{z} f\left(\frac{\partial}{\partial x}\right)\right\|_{h} \leqslant 1$ for all $z \in \mathbb{C}$. Here $h$ stands for some $J$-Hermitian metric on $X$. A $J$-complex line $f$ is said to be a limiting line for $Y$ if $f(\mathbb{C}) \subset \partial Y$ and for every radius $R$ there exists a sequence $f_{n}^{R}: \Delta(R) \rightarrow Y$ of $J$-holomorphic maps of the disk of radius $R$ into $Y$ converging uniformly to $\left.f\right|_{\Delta(R)}$.

Using the Brody reparametrization lemma we can (and we shall always) assume that $\sup \left\{\left\|d_{z} f\left(\frac{\partial}{\partial x}\right)\right\|_{h}: z \in \mathbb{C}\right\}=\left\|d_{0} f\left(\frac{\partial}{\partial x}\right)\right\|_{h}=1$.

An immediate consequence of our theorem and this proposition is the following

Corollary 1. Let $(X, J)$ be a compact almost-complex manifold of dimension 4. Let $D=\cup_{j=1}^{n} D_{j}$ be a reducible $J$ - complex curve, each irreducible component $D_{j}$ of which is an immersed $J$-complex curve. Suppose that for every $j=1, \ldots, n$ the curve $\left(D_{j} \backslash \operatorname{Sing}(D),\left.J\right|_{D_{j}}\right)$ is hyperbolic. Then $(X \backslash D, J)$ is hyperbolically imbedded into $(X, J)$ if and only if there is no $J$-complex line in $X \backslash D$.

By an immersed $J$-complex curve we mean here the image of a $J$-holomorphic map $u: S \rightarrow X$ of a compact Riemann surface $S$, such that $d u$ never vanishes (i.e. , $u(S)$ is a curve without cusps). Let us state one more corollary, which in fact was our initial motivation for considering the existence of complete hyperbolic neighborhoods.

Corollary 2. Let $\mathcal{M}_{\omega, 5 l}$ be the Banach manifold consisting of pairs $\left(J,\left\{D_{j}\right\}_{j=1}^{5}\right)$, where $J$ is any almost-complex structure on $\mathbb{C P}^{2}$ tamed by the Fubini-Studi form $\omega$ and $\left\{D_{j}\right\}_{j=1}^{5}$ the union of five $J$-complex lines in $\mathbb{C P}^{2}$ in general position. The set $\mathcal{H}_{\omega, 5 l}$ consisting of $\left(J,\left\{D_{j}\right\}_{j=1}^{5}\right)$ with $Y=\left(\mathbb{C P}^{2} \backslash \bigcup_{j=1}^{5} D_{j}, J\right)$ hyperbolically imbedded into $\left(\mathbb{C P}^{2}, J\right)$ is an open nonempty subset of $\mathcal{M}_{\omega, 5 l}$.

Nonemptyness of $\mathcal{H}_{\omega, 5 l}$ is the theorem of Bloch. Really, for the standard complex structure on $\mathbb{C P}^{2}$ and standard complex lines $D_{1}, \ldots, D_{5}$ in general position the complex manifold $\mathbb{C P}^{2} \backslash D$ is Kobayashi hyperbolic. Therefore we obtain plenty of examples of almost-complex hyperbolic manifolds of this type (i.e. compact manifold minus a reducible $J$-complex curve). 
We are interested here in the case of almost-complex surfaces, because a generic almost-complex structure $J$ on a manifold of higher dimension does not possess any $J$-complex hypersurface even locally.

We would like also to point out that the following convention is applied throughout this paper (and was already used in this introduction): if we use some statement or definition and refer to the original paper (where this statement/definition is proved/formulated for the integrable case), this means that the same proof/definition goes through also in the non-integrable case and no additional discussion is needed. The reader is supposed to look at the original paper or, if she/he prefers to have everything in one place the book [Ko-2]

The composition of the paper is the following. In $\S 1$ we recall very briefly a few facts from (almost)-complex hyperbolic geometry. In $\S 2$ we state the principal result of this paper, a sort of "gauge invariant Schwarz lemma", and deduce theorem from this lemma. In $\S 3$ we give the proof of this Schwarz lemma. In the last paragraph we give the proofs of Corollaries 1 and 2 and some others results.

\section{Table of Contents}

\section{Introduction.}

1. Kobayashi hyperbolicity of almost-comlex manifolds.

2. Local complete hyperbolicity in $\left(\mathbb{R}^{4}, J\right)$.

3. Proof of the Schwarz-type lemma.

4. Corollaries.

\section{References}

\section{Kobayashi hyperbolicity of almost-complex manifolds.}

Let $(X, J)$ be an almost-complex manifold. For every two sufficiently close points $p$ and $q$ in $X$, there exsists a $J$-holomorphic mapping $u: \Delta \rightarrow X$ such that $u(0)=p$ and $u(a)=q$, where $a \in] 0,1[$ (see [De], Lemma 1$)$. This allows one to define the Kobayashi pseudodistance $k_{X, J}$ on $(X, J)$.

For every point $p \in X$ and every tangent vector $v \in T_{p} X$, there exists for some $R>0$ a (non-unique) $J$-holomorphic map $u: \Delta(R) \rightarrow X$ with $u(0)=p$ and $d_{0} u\left(\frac{\partial}{\partial x}\right)=v$. It is therefore possible to introduce the Royden infinitesimal pseudonorm and prove that the integral pseudometric generated by this pseudonorm coincides with the Kobayashi pseudometric. In the case when this pseudometric is actually a metric (i.e. $(X, J)$ is Kobayashi hyperbolic), this metric induces the given topology on $X$ (by a theorem of Barth [Bt], see also [Kr]).

If $(X, J)$ is not hyperbolic then the result of Brody [Br] states that 


$$
\sup \left\{\left\|d_{0} f(\partial / \partial x)\right\|_{h}: f \in \mathcal{O}_{J}(\Delta ; X)\right\}=\infty,
$$

where $\mathcal{O}_{J}(\Delta ; X)$ denotes the space of $J$-holomorphic maps from the unit disk $\Delta$ to $X$, and $h$ is a $J$-Hermitian metric on $X$.

The proof of the following fundamental result, known as Brody's reparametrization lemma, does not uses the integrability of $J$. Let $(X, J)$ be an almost-complex manifold. Let $f: \Delta(R) \rightarrow X$ be a $J$-holomorphic map with $\left\|d_{0} f\left(\frac{\partial}{\partial x}\right)\right\| \geq c>0$. Then there exists a $J$-holomorphic map $\tilde{f}: \Delta(R) \rightarrow X$ such that

$$
\sup _{z \in \Delta(R)}\left\|d_{z} \tilde{f}(\partial / \partial x)\right\|_{h}\left[\frac{R^{2}-|z|^{2}}{R^{2}}\right]=|| d_{0} \tilde{f}(\partial / \partial x) \|=c .
$$

A combination of (1.1) and (1.2) implies that a compact almost-complex manifold $(X, J)$ is not Kobayashi hyperbolic if and only if it contains a $J$-complex line, see $[\mathrm{De}]$ or $[\mathrm{KrOv}]$ for more details.

\section{Local complete hyperbolicity in $\left(\mathbb{R}^{4}, J\right)$.}

We are going to construct a basis of complete hyperbolic neighborhoods of the origin in $\left(\mathbb{R}^{4}, J\right)$, where $J$ is an arbitrary almost-complex structure of class $C^{1, \alpha}$, $0<\alpha<1$.

There exists a local coordinate system $\left(z_{1}, z_{2}\right)$ in a neighbourhood of 0 such that our almost-complex structure in these coordinates has the form

$$
J\left(z_{1}, z_{2}\right)=\left(\begin{array}{cc}
A\left(z_{1}, z_{2}\right) & 0 \\
0 & B\left(z_{1}, z_{2}\right)
\end{array}\right),
$$

and $J(0)=J_{s t}$, the standard structure of $\mathbb{C}^{2}$. If moreover, a non-singular $J$-complex curve $C \ni 0$ is given, we can arrange that in this coordinate system $C$ is defined by $z_{1}=0$, see $[\mathrm{Sk}]$.

Consider the structure $J_{\varepsilon}\left(z_{1}, z_{2}\right):=J\left(\varepsilon z_{1}, \varepsilon z_{2}\right)$. All we need to prove is that, for $\varepsilon$ small enough, the unit bidisk $\Delta^{2}:=\left\{\left(z_{1}, z_{2}\right):\left|z_{1}\right|<1,\left|z_{2}\right|<1\right\}$ and $\Delta^{2} \backslash\left\{z_{1}=0\right\}$ are complete hyperbolic with respect to $J_{\varepsilon}$. Denote by $z=x+i y$ the coordinate on $\Delta$. Let $f=(u, v) \in \mathcal{O}_{J}\left(\Delta, \Delta^{2}\right)$. The Cauchy-Riemann equation for the first component is

$$
\frac{\partial u}{\partial x}+A(u(z), v(z)) \frac{\partial u}{\partial y}=0,
$$

where $A(u(z), v(z))$ is a $2 \times 2$ real matrix. An obvious transformation gives

$$
\frac{\partial u}{\partial \bar{z}}+(1-A i)^{-1}(1+A i) \frac{\partial u}{\partial z}=0 .
$$

Denote $q_{A}\left(z_{1}, z_{2}\right):=-\left[1-A\left(z_{1}, z_{2}\right) \circ J_{s t}\right]^{-1}\left[1+A\left(z_{1}, z_{2}\right) \circ J_{s t}\right]$. For $z, u, v$ fixed, $q_{A}(z, v, u)$ is an $\mathbb{R}$-linear operator. Let us decompose it into $\mathbb{C}$-linear and $\mathbb{C}$ antilinear part $q_{A}(z, v, u)=\mu^{1}(z, v, u)+\mu^{2}(z, v, u) \circ \sigma$, where $\sigma$ is the conjugation operator. Then the Cauchy-Riemann equation for the first component becomes 


$$
\frac{\partial u}{\partial \bar{z}}-\mu^{1}(z, v, u) \frac{\partial u}{\partial z}-\mu^{2}(z, v, u) \frac{\overline{\partial u}}{\partial z}=0
$$

The second function $v$ will be considered as a parameter. For functions, tensors etc. defined on the open subset $W \subset \mathbb{R}^{N}$, by the $C^{k}(W)$-norm of an object $T$ we shall mean $\|T\|_{C^{k}(W)}:=\sum_{r=0}^{k} \sup \left\{\| D^{r}(T(x) \|: x \in W\}\right.$.

The following Schwarz-type lemma due to Gromov gives us a $C^{1}$-bound on the parameter $v$.

Lemma 2.1. If $J$ is sufficiently close to $J_{\mathrm{st}}$ in $C^{1}$-sense on $\Delta^{2}$, then there exists a constant $C=C\left(\left\|J-J_{\text {st }}\right\|_{C^{1}\left(\Delta^{2}\right)}\right)$ such that every $J$-holomorphic map $f: \Delta \rightarrow \Delta^{2}$ satisfies

$$
\|d f(0)\| \leqslant C
$$

Therefore there exists (another) $C$ such that for every $J$-holomorphic $f=(u, v)$ : $\Delta \rightarrow \Delta^{2}$ we have that $\|u\|_{C^{1}\left(\Delta\left(\frac{1}{2}\right)\right)}$ and $\|v\|_{C^{1}\left(\Delta\left(\frac{1}{2}\right)\right)}$ are bounded by $C$. Rescaling by $z \rightarrow \frac{1}{2} z$ we can suppose that this bound holds everywhere on $\Delta$. Because of the local character of our considerations we can use the standard Euclidean norm $\|\cdot\|$ in all estimates like (2.2).

Suppose a relatively compact domain $D \subset \mathbb{C}$ is given. Fix some universal covering map $\pi: \Delta \rightarrow D$. Suppose $\mu^{1}, \mu^{2} \in C^{1}\left(\mathbb{R}^{6}\right)$ are given and have finite (in the sequel sufficiently small) $C^{1}\left(\mathbb{R}^{6}\right)$-norm. Let us consider the generalized Beltrami equation (2.1) for $C^{1}$-mappings $u: \Delta \rightarrow D$. We suppose that the $C^{1}\left(\mathbb{R}^{2}\right)$ - norm of the parameter $v$ is bounded by some $C$. Further, for a $C^{1}$-map $u: \Delta \rightarrow D$ denote by $u_{\pi}$ any single-valued branch of $\pi^{-1} \circ u$. Denote by $\lambda$ the coordinate on the universal cover $\Delta$. Let also $\varphi_{a}(\lambda)=\frac{a-\lambda}{1-\bar{a} \lambda}$ be the authomorphism of the unit disk interchanging $a \in \Delta$ with the origin. Equation (2.1) rewrites for $u_{a, \pi}:=\varphi_{a} \circ u_{\pi}$ as

$$
\frac{\partial u_{a, \pi}}{\partial \bar{z}}-\mu_{\pi}^{1}\left(z, v, a, u_{a, \pi}\right) \frac{\partial u_{a, \pi}}{\partial z}-\mu_{\pi}^{2}\left(z, v, a, u_{a, \pi}\right) \overline{\left(\frac{\partial u_{a, \pi}}{\partial z}\right)}=0
$$

where $\mu_{\pi}^{1}\left(z, v, a, u_{a, \pi}\right)=\mu^{1}\left(z, v,\left(\pi \circ \varphi_{a}\right)\left(u_{a, \pi}\right)\right)$ and $\mu_{\pi}^{2}\left(z, v, a, u_{a, \pi}\right)=\mu^{2}(z, v,(\pi \circ$ $\left.\varphi_{a}\right)\left(u_{a, \pi}\right) \cdot\left(\overline{\frac{\partial\left(\pi \circ \varphi_{a}\right)}{\partial \lambda}} \cdot\left[\frac{\partial\left(\pi \circ \varphi_{a}\right)}{\partial \lambda}\right]^{-1}\right)$.

In the next paragraph we shall prove the following Schwarz-type lemma: 
Lemma 2.2. There exists an $\varepsilon=\varepsilon(\pi, C)>0$ and a constant $K=K(\pi, C, \varepsilon)<\infty$, such that for every $C^{1}$-solution $w: \Delta \rightarrow \Delta$ of (2.3) with coefficients and parameters satisfying $\left.\left\|\mu^{1,2}\right\|_{C^{1}\left(\mathbb{R}^{6}\right)}\right)<\varepsilon,\|v\|_{C^{1}(\Delta)}<C$ and such that $w(0)=0$, we have the following estimate

$$
\|d w(0)\| \leqslant K
$$

Remark. 1. When one proves the statement, which is now called the SchwarzPick lemma for holomorphic mappings $u: \Delta \rightarrow \Delta$, one proves in fact two statemets. Firstly, the Schwarz lemma, which gives the universal bound for the absolute value of the derivative of $u$ provided $u(0)=0$, i.e. , one obtains $|d u(0)| \leqslant 1$. Second, using "the gauge invariance" of the homogeneous Cauchy-Riemann equation with respect to the "gauge group" $G:=A u t_{H o l}(\Delta)$, one obtains the "gauge invariant" form of this statement:

$$
\frac{|d u(0)|}{1-|u(0)|^{2}} \leqslant 1,
$$

which means exactly the completeness of Kobayashi metric on the unit disk.

The generalized Cauchy-Riemann (or Beltrami) equation (2.1) has no symmetries except for very special $\mu$ 's. Therefore, instead of considering only one equation we "symmetrize" the problem and consider the whole family of equations (2.3) with a parameter $\varphi_{a} \in G$, our gage group. Uniform estimate (2.4) of the derivative at zero allows us to make the same conclusions as for the standard Cauchy-Riemann equation, because the problem is now "enforced" with nessessary symmetries.

2. However the price for this is that the coefficients of (2.4) are no longer bounded in any Sobolev or Hölder space as the parameter $a \rightarrow \partial \Delta$. They are bounded in the sup-norm only, and this is clearly not sufficient to bound the derivative at zero. The trick is (see Step 1 of the proof in the next paragraph) that there is an a priori bound for the solutions $w$ of (2.4) satisfying $w(0)=0$ in the Hölder norm on a neighborhood of zero. This allows us to control the Hölder norm of the coefficients and apply elliptic bootstrap one more time (see Step 2).

Corollary 2.3. For $J$ sufficiently $C^{1}$-close to $J_{\text {st }}$, the open set $\left(\Delta^{2}, J\right)$ is complete hyperbolic.

Proof. As we have already explained, by taking $J$ close to $J_{\text {st }}$ we can bound the components $(u, v)$ of a $J$-holomorphic map $f: \Delta \rightarrow \Delta^{2}$ using Gromov's lemma. The explicit form of $q_{A}$ and consequently of $\mu^{1,2}$ shows that, again by taking $J$ close to $J_{\text {st }}$, we can ensure that $\left\|\mu^{1,2}\right\|_{C^{1}}<\varepsilon$ for the $\varepsilon$ from Lemma 2.2 applied to the identity covering $\pi=\mathrm{Id}: \Delta \rightarrow \Delta$. Let $u(0)=a$. Applying Lemma 2.2 to $w=\varphi_{a} \circ u$, we obtain that $\|d w(0)\| \leq K$, where $K$ does not depend on $w$. 
But $d w(0)=\frac{1}{|a|^{2}-1} \cdot d u(0)$ and therefore, for every solution $u: \Delta \mapsto \Delta$ of $(2.1)$ such that $u(0)=a$, one has $\frac{1}{\|d u(0)\|} \geq \frac{1}{K\left(1-|a|^{2}\right)}$. This means that, for every vector $(\xi, 0)$ in $T_{(a, b)} \Delta^{2}$, we have $k_{\Delta^{2}, J}((a, b),(\xi, 0)) \geq \frac{C_{1}|\xi|}{1-|a|^{2}}$, by the definition of the Kobayashi-Royden pseudometric.

The same considerations apply, of course, to the second component of $(u, v)$. This implies that $k_{\Delta^{2}, J}((a, b),(0, \eta)) \geq \frac{C_{2}|\eta|}{1-|b|^{2}}$. Therefore,

$$
k_{\Delta^{2}, J}((a, b),(\xi, \eta)) \geq \max \left(\frac{C_{1}|\xi|}{1-|a|^{2}}, \frac{C_{2}|\eta|}{1-|b|^{2}}\right),
$$

which gives us the desired conclusion.

Corollary 2.4. For $J$ sufficiently $C^{1}$-close to $J_{\text {st }}$ on the whole bidisk $\Delta^{2}$, the open set $\left(\Delta^{2} \backslash(\{0\} \times \Delta), J\right)$ is complete hyperbolic.

Proof. Let us apply Lemma 2.2 to the universal covering $\pi: \Delta \rightarrow \Delta^{*}=D$ given by $\pi(\lambda)=\exp \frac{\lambda-1}{\lambda+1}$. For a solution $u: \Delta \rightarrow \Delta^{*}$ of $(2.1)$, set $a=u(0)$ and $b=\ln (a)$ with imaginary part $\operatorname{Im}(b) \in\left[-\pi, \pi\left[\right.\right.$. Let also $c=\frac{b+1}{1-b} \in \Delta$. Therefore $c$ is one of the preimages of $a$ under the covering map $\pi$. Take the single-valued branch $u_{\pi}$ of $\pi^{-1} \circ u$ such that $u_{\pi}(0)=c$, and consider $w=\varphi_{c} \circ u_{\pi}$, a solution of (2.3). Lemma 2.2 gives us that $\|d w(0)\| \leqslant K$, where $K$ does not depend on anything involved. But direct computation shows that

$$
d w(0)=\frac{|c|^{2}-1}{\left(1-|c|^{2}\right)^{2}} \frac{2}{(1+b)^{2}} \frac{d u(0)}{a}=\frac{1}{|c|^{2}-1} \cdot \frac{2}{(1+b)^{2}} \frac{d u(0)}{a} .
$$

Therefore

$$
d w(0)=\frac{1}{\left|\frac{1+\ln a}{1-\ln a}\right|^{2}-1} \frac{2}{(1+\ln a)^{2}} \frac{d u(0)}{a},
$$

and hence

$$
\frac{1}{|d u(0)|} \geq K_{1} \frac{1}{|a| \ln \frac{1}{|a|}}
$$

for $a$ close to zero. By the definition of the Kobayashi-Royden pseudometric, for every vector $(\xi, 0)$ in $T_{(a, b)}\left(\Delta^{2} \backslash \Delta^{*}\right)$, we have

$$
k_{\Delta^{2} \backslash \Delta^{*}, J}((a, b),(\xi, 0)) \geq K_{1} \frac{1}{|a| \ln \frac{1}{|a|}}
$$

for $a$ close to zero, and therefore, $k_{\Delta^{2} \backslash \Delta^{*}, J}((a, b),(\xi, \eta))=\bigcirc\left(\frac{1}{|a| \ln \frac{1}{|a|}}\right) \sqrt{\xi^{2}+\eta^{2}}$. The consequence is that every path leading to 0 has infinite length, which proves the corollary. 


\section{$\S 3$. Proof of the Schwarz-type lemma.}

Proof. Suppose that the conclusion of the lemma is not true. Let $\left(h_{n}\right)$ be a sequence of mappings $h_{n}: \Delta \mapsto \Delta, h_{n}(0)=0$, solutions of $(2.3)$, such that $\left|d h_{n}(0)\right| \rightarrow \infty$. For each $n$, denote by $v_{n}$ the corresponding functional parameter and by $a_{n}$ the corresponding parameter in $\Delta$.

Step 1. There exists $\delta>0$ and $n_{0}$ such that, for $n \geqslant n_{0} h_{n} \in C^{0, \frac{1}{2}}\left(\Delta_{\delta}, \Delta_{\frac{1}{2}}\right)$ and $\left\{h_{n}\right\}_{n \geqslant n_{0}}$ is a bounded set in this space.

Consider a test function $\rho$ which is $C^{\infty}$ and such that $\rho \equiv 1$ on $\Delta_{\frac{3}{4}}$ and $\rho \equiv 0$ outside $\Delta$, and denote $h_{n}^{\rho}=\rho h_{n}$. The equation (2.3) for $h_{n}$, written in terms of $h_{n}^{\rho}$, gives

$$
\begin{gathered}
\frac{\partial h_{n}^{\rho}}{\partial \bar{z}}-\mu_{v, a_{n}}^{1}\left(z, h_{n}\right) \frac{\partial h_{n}^{\rho}}{\partial z}-\mu_{v, a_{n}}^{2}\left(z, h_{n}\right) \overline{\left(\frac{\partial h_{n}^{\rho}}{\partial z}\right)}=h_{n} \frac{\partial \rho}{\partial \bar{z}}-h_{n} \mu_{v, a}^{1}\left(z, h_{n}\right) \frac{\partial \rho}{\partial z}- \\
-\bar{h}_{n} \mu_{v, a}^{2}\left(z, \overline{\left.h_{n}\right) \overline{\left(\frac{\partial \rho}{\partial z}\right)} .}\right.
\end{gathered}
$$

Consider the Cauchy-Green operator $T_{C G}: L^{4}(\Delta) \rightarrow L^{1,4}(\Delta)$ and the CalderonZygmund operator $T_{C Z}: L^{4}(\Delta) \rightarrow L^{4}(\Delta)$, defined respectively as

$$
T_{C G} g(z):=\frac{1}{2 i \pi} \iint_{\Delta} \frac{g(\zeta)}{\zeta-z} d \zeta \wedge d \bar{\zeta}
$$

and

$$
T_{C Z} g(z):=p . v \cdot \frac{1}{2 i \pi} \iint_{\Delta} \frac{g(\zeta)}{(\zeta-z)^{2}} d \zeta \wedge d \bar{\zeta}
$$

$T_{C G}$ and $T_{C Z}$ are bounded linear operators in Sobolev and Hölder spaces. Let us denote by $C_{k, p}$ the norm of $T_{C G}: L^{k, p}(\Delta) \rightarrow L^{k+1, p}(\Delta)$ and by $C_{k, \alpha}$ the norm of $T_{C G}: C^{k, \alpha} \rightarrow C^{k+1, \alpha}$. By $A_{k, p}$ and $A_{k, \alpha}$ we denote the similar constants for $T_{C Z}: L^{k, p} \rightarrow L^{k, p}$ and $T_{C Z}: C^{k, \alpha} \rightarrow C^{k, \alpha}$. Here always $k \geqslant 0,1<p<\infty$ and $0<\alpha<1$.

We know that $\bar{\partial} \circ T_{C G}=I d$ and that $T_{C Z}=\partial \circ T_{C G}$. We also know that on the closure in $L^{1,2}$ of the space of compactly supported $C^{\infty}$ maps, $T_{C Z} \circ \bar{\partial}=\partial$ and $T_{C G} \circ \bar{\partial}=I d$. We remark that the $h_{n}^{\rho}$ are in this space. Set $g_{1}=\frac{\partial \rho}{\partial \bar{z}}-\mu_{v, a}^{1}\left(z, h_{n}\right) \frac{\partial \rho}{\partial z}$ and $g_{2}=-\mu_{v, a}^{2}\left(z, h_{n}\right) \overline{\left(\frac{\partial \rho}{\partial z}\right)}$. The equation (3.1) can be written as

$$
\left[I d-\mu_{v, a}^{1}\left(., h_{n}\right) \circ T_{C Z}-\mu_{v, a}^{2}\left(., h_{n}\right) \circ \sigma \circ T_{C Z}\right] \frac{\partial}{\partial \bar{z}} h_{n}^{\rho}=h_{n} g_{1}+\bar{h}_{n} g_{2}
$$

We remark that $h_{n} g_{1}+\bar{h}_{n} g_{2}$ is a bounded sequence in $L^{4}\left(\Delta, \mathbb{R}^{2}\right)$. Indeed, $h_{n}$ takes its values in $\Delta$ and $g_{1}$ and $g_{2}$ are bounded. Since $\left\|T_{C Z}\right\|_{L^{4}}$ is bounded, and as $\mu^{1}$ and $\mu^{2}$ are as small as we want, this operator is invertible. Therefore, 


$$
\frac{\partial}{\partial \bar{z}} h_{n}^{\rho}=\left[I d-\mu_{v, a}^{1}\left(., h_{n}\right) \circ T_{C Z}-\mu_{v, a}^{2}\left(., h_{n}\right) \circ \sigma \circ T_{C Z}\right]^{-1}\left(h_{n} g_{1}+\bar{h}_{n} g_{2}\right)
$$

and hence

$$
h_{n}^{\rho}=T_{C G}\left(\left[I d-\mu_{v, a}^{1}\left(., h_{n}\right) \circ T_{C Z}-\mu_{v, a}^{2}\left(., h_{n}\right) \circ \sigma \circ T_{C Z}\right]^{-1}\left(h_{n} g_{1}+\bar{h}_{n} g_{2}\right)\right) \text {. }
$$

Thus $\left(h_{n}^{\rho}\right)$ is a bounded sequence in $L^{1,4}(\Delta, \Delta)$. By Sobolev imbedding $L^{1, p} \subset$ $C^{0, \alpha=\frac{2}{p}}$, our sequence $\left(h_{n}^{\rho}\right)$ is bounded in $C^{0, \frac{1}{2}}$. Therefore, $\left(h_{n}^{\rho}\right)$ is a bounded and equicontinuous family of mappings on compacts in $\Delta$. From the theorem of Ascoli we deduce that it is compact, and extract from it a converging subsequence. Therefore, $\left(h_{n}\right)$ converges uniformly on $\Delta_{\frac{3}{4}}$ to a mapping $h: \Delta_{\frac{3}{4}} \rightarrow \Delta$ such that $h(0)=0$. There exists $\delta$ such that $h\left(\Delta_{\delta}\right) \subset \Delta_{\frac{1}{2}}$ and consequently $h_{n}\left(\Delta_{\delta}\right) \subset \Delta_{\frac{1}{2}}$ for $n$ big enough. Therefore $h_{n}$ is bounded in $C^{0, \frac{1}{2}}\left(\Delta_{\delta}, \Delta_{\frac{1}{2}}\right)$ starting from some $n>>1$.

Step 2. There exists $n_{1}$ such that $h_{n} \in C^{1, \frac{1}{2}}\left(\Delta_{\frac{\delta}{2}}\right)$ for $n \geqslant n_{1}$ and $\left\{h_{n}\right\}_{n \geqslant n_{0}}$ is a bounded set in this space.

Parameters $v_{n}$ are bounded in $C^{1}(\Delta)$, and $h_{n} \in C^{0, \frac{1}{2}}\left(\Delta_{\delta}, \Delta_{\frac{1}{2}}\right)$. Therefore $\mu_{v, a_{n}}^{1}\left(z, h_{n}\right)$ and $\mu_{v, a_{n}}^{2}\left(z, h_{n}\right)$ are bounded in $C^{0, \frac{1}{2}}\left(\Delta_{\delta}\right)$. Put $f_{n}(z):=h_{n}(\delta z)$. This is a bounded sequence in $C^{0, \frac{1}{2}}(\Delta, \Delta)$. All we need to prove is that $\left\{f_{n}\right\}$ is bounded in $C^{1, \frac{1}{2}}\left(\Delta_{\frac{1}{2}}\right)$. We see that $f_{n}$ satisfy

$$
\frac{\partial f_{n}}{\partial \bar{z}}-\mu_{v_{n}, a_{n}}^{1}\left(z, f_{n}(z)\right) \frac{\partial f_{n}}{\partial z}-\mu_{v_{n}, a_{n}}^{2}\left(z, f_{n}\right) \overline{\left(\frac{\partial f_{n}}{\partial z}\right)}=0
$$

with $\mu_{v_{n}, a_{n}}^{1}\left(z, f_{n}\right)$ and $\mu_{v_{n}, a_{n}}^{2}\left(z, f_{n}\right)$ uniformly bounded in $C^{0, \frac{1}{2}}(\Delta, \Delta)$. We can repeat now the reasonings from Step 1 applied to $f_{n}$ instead of $h_{n}$ and arrive at equation (3.2) but considered in Hölder spaces. In this way we get the boundedness of $f_{n}$ in $C^{1, \frac{1}{2}}\left(\Delta_{\frac{1}{2}}\right)$. Contradiction.

\section{Corollaries.}

We shall prove Corollaries 1 and 2 from the Introduction first . The crucial point in the proof is the following statement about the positivity of intersections of $J$-complex curves due to $[\mathrm{M}-\mathrm{W}]$.

Theorem. Let $u_{i}: \Delta \rightarrow\left(\mathbb{R}^{4}, J\right), i=1,2$ be two primitive distinct $J$-complex disks such that $u_{1}(0)=u_{2}(0)=0$. Set $M_{i}:=u_{i}(\Delta)$. Let $Q=M_{1} \cap M_{2}$ be the intersection set of the disks. If $J$ is $C^{1}$-smooth, then the following is true: 
i) The set $\left\{\left(z_{1}, z_{2}\right) \in \Delta \times \Delta: u_{1}\left(z_{1}\right)=u_{2}\left(z_{2}\right)\right\}$ is a discrete subset of $\Delta \times \Delta$. In particular, $u_{1}(\Delta) \cap u_{2}(\Delta)$ is a countable set. If moreover $u_{1}(\partial \Delta) \cap u_{2}(\partial \Delta)=\varnothing$ then $u_{1}(\Delta) \cap u_{2}(\Delta)$ is finite.

ii) The intersection index $\delta_{p}$ of $M_{1}$ and $M_{2}$ at any such point $p \in Q$ is strictly positive. Moreover, if $\mu_{1}$ and $\mu_{2}$ are the multiplicities of $u_{1}$ and $u_{2}$ in $z_{1}$ and $z_{2}$, respectively, with $u_{1}\left(z_{1}\right)=u_{2}\left(z_{2}\right)=p$, then the intersection number $\delta_{p}$ at $p=u_{j}\left(s_{j}\right)$ is at least $\mu_{1} \cdot \mu_{2}$;

iii) $\delta_{p}=1$ iff $M_{1}$ and $M_{2}$ intersect at $p$ transversally.

For a proof see Theorems 7.1 and 7.3 in [M-W], or Theorem 3.5.1 in [I-S].

We shall need the following topological description of the total intersection number $\delta=\sum_{p \in Q} \delta_{p}$ of $M_{1}$ and $M_{2}$.

Take a sphere $S_{r}$ of radius $r$ around the origin, which bounds the ball $B_{r}$. Then, for $r$ small enough, the intersections $M_{i} \cap S_{r}$ are transversal, and moreover, since $Q$ is at most countable, we can find $r>0$ as small as we wish with $\gamma_{1}^{r} \cap \gamma_{2}^{r}=\varnothing$, where $\gamma_{i}^{r}=M_{i} \cap S_{r}$. Moreover, $\gamma_{i}^{r}$ will be transversal to the distribution $F_{r}$ of $J$-complex tangent planes to $S_{r}$, see Lemma A2.1.1 in [I-S]. Let $l\left(\gamma_{1}^{r}, \gamma_{2}^{r}\right)$ denote the linking number of the curves $\gamma_{1}^{r}$ and $\gamma_{2}^{r}$. A standard lemma from topology (see e.g. [Rf]) says now that

$$
l\left(\gamma_{1}^{r}, \gamma_{2}^{r}\right)=\sum_{p \in Q \cap B_{r}} \delta_{p} .
$$

The positivity of intersections of $J$-complex curves implies that if $M_{1}$ and $M_{2}$ do intersect at zero, then this linking number is not zero (and is positive).

Proof of Corollary 1. All we need to check is that $Y=X \backslash D$ does not admit a limiting $J$-complex line. Let $u: \mathbb{C} \rightarrow D$ be a limiting $J$-complex line for $Y=X \backslash D$ and let $u(\mathbb{C}) \subset D_{1}$ for definitivity. $u(\mathbb{C})$ cannot be contained in $D_{1} \backslash \operatorname{Sing}(D)$, because this curve is assumed to be hyperbolic. Therefore there exists $z_{0} \in \mathbb{C}$ such that $p:=u\left(z_{0}\right) \in D_{1} \cap \operatorname{Sing}(D)$. Take as $M_{1}$ the piece of $D_{1}$ which containing the image of the germ of $u$ at $z_{0}$ and as $M_{2}$ a germ of any other branch of some $D_{i}$ passing through $p$.

Chose a sphere $S_{r}$ as above. Take some $R$ such that $\Delta(R) \supset u^{-1}\left(M_{1}\right)$. Let $u_{n}: \Delta(R) \rightarrow Y$ be $J$-holomorphic mapping approaching uniformly to $u$. Then for $n>>1$ the linking number $l\left(\gamma_{n}, \gamma_{2}^{r}\right)$ is the same as $l\left(\gamma_{1}^{r}, \gamma_{2}^{r}\right)$. Here $\gamma_{n}$ is an appropriate component of $u_{n}(\Delta(R)) \cap S_{r}$. The positivity of $l\left(\gamma_{1}^{r}, \gamma_{2}^{r}\right)$ implies now the positivity of $l\left(\gamma_{n}, \gamma_{2}^{r}\right)$ for $n$ large enough. This contradicts the definition of a limiting line, i.e.,$u_{n}(\mathbb{C})$ should not intersect $D$. 
Proof of Corollary 2. This is rather similar to the previous one. Suppose we can find a sequence $\left(\left\{D_{i}^{(k)}\right\}_{i=1}^{5}, J_{k}\right)$ in $\mathcal{M}_{\omega, 5 l}$, such that $Y_{k}=\left(\mathbb{C P}^{2} \backslash D^{(k)}, J_{k}\right)$ are not hyperbolic and converge to $\left(\left\{D_{i}\right\}_{i=1}^{5}, J\right)$ in $\mathcal{M}_{\omega, 5 l}$, such that $Y=\left(\mathbb{C P}^{2} \backslash D, J\right)$ is hyperbolic. Take $J_{k}$ and $J$-Hermitian metrics $\omega\left(\cdot, J_{k} \cdot\right)$ and $\omega(\cdot, J \cdot)$ on $\mathbb{C P}^{2}$. By Corollary 1 there exist $J_{k}$-complex line $u_{k}: \mathbb{C} \rightarrow Y_{k}$. Gradients of $u_{k}$ are uniformly bounded and therefore some subsequence, stil denoted by $u_{k}$, converges uniformly on compacts in $\mathbb{C}$. All topologies on the space of holomorphic mappings are equivalent (see e.g. Corollary 3.2.2 from [I-S]) and therefore $u_{k}$ converge to some $J$-holomorphic $u: \mathbb{C} \rightarrow \mathbb{C P}^{2}$ in the $C^{1}$-sense. Since $\|d u(0)\|_{h}=\lim _{k \rightarrow \infty}\left\|d u_{k}(0)\right\|_{h_{k}}=\frac{1}{2}$, the map $u$ is a $J$-complex line. $u(\mathbb{C})$ cannot be contained in $Y$ because of the hyperbolicity of the latter. So $u(\mathbb{C}) \cap D \neq \varnothing$.

Considerations similar to that from the proof of Corollary 1 show that in this case $u_{k}(\mathbb{C}) \cap D^{(k)} \neq \varnothing$ for $k>>1$. Contradiction.

A similar proof yields one more corollary. Denote by $\mathcal{M}_{\omega, d}$ the Banach manifold of pairs $(J, D)$, where $J$ is an $\omega$-tamed a.-c. structure on $\mathbb{C P}^{2}$ and $D$ is some irreducible $J$-complex curve of degree $d$ in $\mathbb{C P}^{2}$. Denote by $\mathcal{H}_{\omega, d}$ the subset of $(J, D) \in \mathcal{M}_{\omega, d}$ such that $\left(\mathbb{C P}^{2} \backslash D, J\right)$ is hyperbolically imbedded into $\left(\mathbb{C P}^{2}, J\right)$.

Corollary 4.1. The set $\mathcal{H}_{\omega, d}$ is a nonempty open subset of $\mathcal{M}_{\omega, d}$, provided $d \geqslant 5$.

$\mathcal{H}_{\omega, d}$ is not empty by the result of Zaidenberg [Za-2], which asserts that there exists an open set in the manifold of algebraic curves of degree $d \geqslant 5$ in $\mathbb{C P}^{2}$ such that $\mathbb{C P}^{2} \backslash D$ is hyperbolically imbedded into $\mathbb{C P}^{2}$ for every curve $D$ from this set .

\section{R E F E R E N C E S}

[Bt] T.J. BARTh.: The Kobayashi distance induces the standard topology. Proc. Amer. Math. Soc. 35, 439-440 (1972).

[Br] R. Brody.: Compact manifolds and hyperbolicity. Trans. Amer. Math. Soc. 235 213-219 (1978).

[De] R. Debalme.: Kobayashi hyperbolicity of almost complex manifolds. Preprint of the university of Lille, IRMA 50 (1999), math.CV/9805130.

[Gr] M. GreEn.: The hyperbolicity of the complement of $2 n+1$ hyperplanes in general position in $\mathbb{P}^{n}$, and related results. Proc. Amer. Math. Soc. 66 109-113 (1977).

[G] M. Gromov.: Pseudo holomorphic curves in symplectic manifolds. Invent. math. 82 307-347 (1985).

[I-S] S. Ivashrovich, V. Shevchishin.: Complex Curves in AlmostComplex Manifolds and Meromorphic Hulls. Lecture Notes in Schriftenreihe des Graduiertenkollegs, Geometrie und Math. Physik, Ruhr-Uni-Bochum, Heft 36, 1-186 (1999), see also math.CV/9912046. 
[Ki] P. Kiernan.: Hyperbolically Imbedded Spaces and the Big Picard Theorem. Math. Ann. 204 203-209 (1973).

[Ko-1] S. Kobayashi.: Invariant distances on complex manifolds and holomorphic mappings. J. Math. Soc. Japan 19 460-480 (1967).

[Ko-2] S. Kobayashi.: Hyperbolic Complex Spaces. Springer (1998).

[Kr] B. Kruglikov.: On the Kobayashi-Royden pseudonorm for almost complex manifolds. math. dg-ga/9708012.

[KrOv] B. Kruglikov and M. Overholt.: The Kobayashi pseudodistance on almost complex manifolds. Preprint of the university of Tromsoe 19 (1997) math. dg-ga/9703005.

[La] S. LANG.: Introduction to complex hyperbolic spaces. Springer-Verlag New York (1987).

[McD] D. McDuff.: Singularities and positivity of intersections of $J$ holomorphic curves. Holomorphic curves in symplectic geometry, eds. M. Audin and J.Lafontaine, Birkhauser, Basel 191-215 (1994).

[M-W] M. Micallef , B. White The structure of branch points in minimal surfaces and in pseudoholomorphic curves. Ann. Math. 139 35-85 (1994).

[Rf] Rolfsen, D.: Knots and links. Publish or perish, N7, (1976).

[Ro] H.L. Royden.: Remarks on the Kobayashi metric. Springer LNM 185 125-137 (1970).

[Sk] J.-C. Sikorav.: Some properties of holomorphic curves in almost complex manifolds. Holomorphic curves in symplectic geometry, eds. M. Audin and J.Lafontaine, Birkhauser, Basel 165-189 (1994).

[Za-1] M. Zaidenberg.: Picard's Theorem and Hyperbolicity. Siberian Math. J., 24 858-867 (1983).

[Za-2] M. Zaidenberg.: Stability of hyperbolic embeddedness and construction of examples. Math. USSR Sbornik, 63, N 2, 351-361 (1989). 